Vol 3, Issue 2, 2021 (88-77)
http://journal.unpad.ac.id/idjp

\title{
Development of a quantitative method for sunitinib N-oxide using LC-MS/MS
}

\author{
Yuya Ishikawa ${ }^{1,2}$, Takuya Araki, Ph.D. ${ }^{1,2}$, Miki Takenaka Sato, Ph.D. ${ }^{1,2,3}$, Hideaki \\ Yashima, Ph.D. ${ }^{2}$, Daisuke Nagano, Ph.D. ${ }^{1}$, Koujirou Yamamoto, Ph.D. ${ }^{1,2}$ \\ ${ }^{1}$ Department of Clinical Pharmacology and Therapeutics, Gunma University Graduate School \\ of Medicine, 3-39-22 Showa-machi, Maebashi, Gunma 371-8511, Japan \\ ${ }^{2}$ Department of Pharmacy, Gunma University Hospital, 3-39-15 Showa-machi, Maebashi, \\ Gunma 371-8511, Japan \\ ${ }^{3}$ Division of Pharmacotherapeutics, Department of Clinical Pharmacy, Showa University \\ School of Pharmacy, 1-5-8 Hatanodai, Shinagawa-ku, Tokyo 142-8555, Japan
}

\author{
Submitted : 24 December 2021, Revised : 12 January 2022, Accepted : 13 January 2022
}

\begin{abstract}
We developed a simple method for quantifying sunitinib N-oxide (SNO) in human serum using a supported liquid extraction (SLE) method and liquid chromatography/tandem mass spectrometry (LC-MS/MS) to assess the impact of SNO on adverse drug reactions (ADRs) caused by sunitinib. SNO was extracted using an SLE method and analyzed using an XevoTQ (Waters) LC-MS/MS system. SNO and voriconazole (internal standard; ISTD) were detected in ESI positive mode, with transitions at 415.4/326.3 for SNO and 350.1/281.1 for voriconazole. The retention times of SNO and voriconazole were 2.25 and $2.67 \mathrm{~min}$, respectively, and good calibration curve was obtained from $0.1-5.0 \mathrm{ng} / \mathrm{mL}$ for SNO. The regression equation (weight $=1 / \mathrm{x} 2$ ) describing the calibration curve in human serum was $\mathrm{y}=$ $2.81 \times 10-9 \times 2+0.000253 \mathrm{x}-0.00202(\mathrm{R} 2=0.990)$, where $\mathrm{y}$ is the peak area ratio of SNO against the ISTD and $\mathrm{x}$ is the nominal concentration of SNO. The intra- and inter-assay accuracy varied between -2.4 and $15.6 \%$ and all data except the limit of quantification (LOQ) were within $\pm 10 \%$. The precision varied between $6.7-15.4 \%$ and all data except LOQ were under $15 \%$. The mean recovery ratio of SNO was $90.3 \pm 4.9 \%$, and the mean matrix factor was $0.96 \pm 0.031$. This is the first report of a method to quantify SNO in blood. This method will help in elucidating the effects of SNO in humans, contribute to the elucidation of the ADRs expression factors associated with sunitinib, and aid in optimizing treatment with sunitinib.
\end{abstract}

Keywords: sunitinib N-oxide, metabolite, adverse drug reaction, hand foot skin reaction 


\section{Introduction}

Sunitinib malate is a multi-target tyrosine kinase inhibitor (TKI) and is used as an oral drug for treating renal cell carcinoma, gastrointestinal stromal tumor, and pancreatic neuroendocrine tumor [1-4]. Sunitinib strongly inhibits multiple tyrosine kinases, including VEGFRs 1-3 and PDGFRs $\alpha$ and $\beta$, and exhibits high anticancer efficacy [4]. However, the therapeutic efficacy of sunitinib varies greatly among patients, and adverse drug reactions (ADRs) such as hand foot skin reaction (HFSR), a characteristic ADR of sunitinib, force some patients to abandon treatment despite high clinical efficacy $[5,6]$. Studies of the inter-individual differences of the clinical efficacies of TKIs include detailed investigations of the influence of drug concentration on the clinical efficacy of sunitinib, with many studies showing efficacy depending on the drug concentration [7-9]. In addition, sunitinib is metabolized by CYP3A4 to $\mathrm{N}$-desethyl sunitinib, which exhibits tyrosine kinase inhibitory activities similar to sunitinib. Thus, the sum of the concentration of sunitinib and of $\mathrm{N}$-desethyl sunitinib, and not the concentration of sunitinib alone, is likely important in predicting the efficacy of sunitinib [10]. The clinical usefulness of dosing designs based on the total sunitinib concentration has been reported in several papers[7-9].

Although the expression of some ADRs in individuals, such as decreased platelet count, has been reported to depend on the sum of the concentration of sunitinib and of $\mathrm{N}$-desethyl sunitinib in blood, many ADRs such as HFSR, bleeding, and hypertension are reported to not depend on either the blood sunitinib concentration or the sum of the concentration of sunitinib and of $\mathrm{N}$ desethyl sunitinib [9]. The genetic polymorphism of $\mathrm{ABCG} 2$, a transporter involved in the extracellular excretion of sunitinib, has been reported to have a significant effect on the expression of $\mathrm{ADR}$, and the frequency of ADR expression in patients with ABCG2 rs2231142 $\mathrm{CC}$ is high [11]. However, ADRs that interfere with the continuation of treatment occur with a certain frequency even in patients with wild type ABCG2 [12], demonstrating that the risk and cause of ADRs remain insufficiently evalu ated.

The factors causing ADR were recently studied by analysis of the metabolites of drugs, and several studies detected the causative factors [9, 13-15]. For example, in sorafenib, classified as a TKI as is sunitinib, the area under the curve of the $\mathrm{N}$ oxide has been reported to be significantly higher in a group of patients that required a reduced dosage due to ADRs [16], and therefore the $\mathrm{N}$-oxide form is attracting attention as a candidate causative agent for ADRs caused by sorafenib.

Previously, we comprehensively analyzed the metabolites of sunitinib in the blood of patients taking sunitinib to search for the factors causing ADRs due to sunitinib and reported that sunitinib $\mathrm{N}$ oxide, a photodegradant of sunitinib, was detected in the blood of patients taking sunitinib [17]. Since HFSR caused by sunitinib has been reported to be diminished by the administration of antioxidants [18], we considered that sunitinib $\mathrm{N}$-oxide may affect the expression of ADRs such as HFSR. However, currently there is no method for measuring the blood concentration of sunitinib $\mathrm{N}$ oxide, and the impact of sunitinib $\mathrm{N}$-oxide on the expression of ADRs has not been assessed. So, in this study, we developed a method for measuring the concentrations of 
sunitinib $\mathrm{N}$-oxide in blood to clarify the impact of sunitinib N-oxide on the expression of ADRs of sunitinib.

To date, many methods using liquid chromatography with ultraviolet detection (LC-UV) or liquid chromatography-tandem mass spectrometry (LC-MS/MS) have been reported as methods for analyzing sunitinib and $\mathrm{N}$-desethyl sunitinib [19-21]. However, since the concentration of sunitinib $\mathrm{N}$-oxide was estimated to be about $1 / 10$ to $1 / 20$ that of sunitinib in our previous study [17], an analytical method that is more sensitive than that used for quantifying sunitinib is required to study the effect of sunitinib $\mathrm{N}$ oxide on the body. Therefore, in this study, we used a supported liquid extraction (SLE) pretreatment method to concentrate thesample and analyzed sunitinib and its metabolites in blood using LC-MS/MS

\section{Materials and Methods}

\subsection{Materials}

Sunitinib (>99\% purity) was purchased from LC Laboratories (Woburn, MA, USA), sunitinib N-oxide (>99\% purity) and $\mathrm{N}$-desethyl sunitinib (96\% purity) were purchased from Toronto Research Chemicals (Toronto, Canada) and voriconazole ( $>98 \%$ purity) was purchased from Tokyo Chemical Industry Co., Ltd. (Tokyo, Japan). ISOLUTE SLE $+400 \mu \mathrm{L}$ 96-well plates (SLE array plates) were purchased from Biotage Japan Ltd. (Tokyo, Japan). All other reagents were obtained from commercial sources and were of LCMS, HPLC, or special grade.

\subsection{Preparation of stock solutions, working solutions, calibration samples, and quality control samples}

Primary stock solutions of $1.0 \mathrm{mg} / \mathrm{mL}$ of sunitinib $\mathrm{N}$-oxide, sunitinib, $\mathrm{N}$-desethyl sunitinib, and voriconazole, used as an internal standard (ISTD), were separately prepared in methanol. The primary stock solution of mixture of sunitinib $\mathrm{N}$-oxide, sunitinib, and $\mathrm{N}$-desethyl sunitinib was diluted with $50 \%$ methanol to yield standard working solutions $(0.5,1.25,2.5$, $5,12.5,25,50,125,250,500$, and 1250 $\mathrm{ng} / \mathrm{mL})$. The primary stock solution of the ISTD was diluted in 50\% methanol to 1 $\mu \mathrm{g} / \mathrm{mL}$ or $100 \mathrm{ng} / \mathrm{mL}$. The stock solutions and other diluted solutions were stored at $20^{\circ} \mathrm{C}$ and $4^{\circ} \mathrm{C}$, respectively, under dark conditions. All solutions were equilibrated to room temperature before use. Calibration samples and quality control (QC) samples were prepared by spiking blank pooled human serum (PHS) with a given volume of different working solutions. The concentrations of sunitinib $\mathrm{N}$-oxide for the calibration sample of sunitinib $\mathrm{N}$-oxide were set at $0.1,0.25,0.5,1,2.5$, and 5 $\mathrm{ng} / \mathrm{mL}$. Calibration samples for sunitinib and $\mathrm{N}$-desethyl sunitinib were prepared at 7 concentrations: $2.5,5,10,25,50,100$, and $250 \mathrm{ng} / \mathrm{mL}$. QC samples of sunitinib Noxide as the lower limit of quantitation, low level, middle level, and high level were set as $0.1,0.25,1$, and $5 \mathrm{ng} / \mathrm{mL}$, respectively, and those of sunitinib and N-desethyl sunitinib were set as 2.5, 5, 50, and 250 $\mathrm{ng} / \mathrm{mL}$, respectively.

\subsection{Sample preparation}

The serum samples for analysis of sunitinib $\mathrm{N}$-oxide were treated using an SLE array plate. Briefly, a mixture of $50 \mu \mathrm{L}$ of blank PHS, $10 \mu \mathrm{L}$ of a standard working solution, $10 \mu \mathrm{L}$ of the ISTD $(100 \mathrm{ng} / \mathrm{mL})$, and $330 \mu \mathrm{L}$ of $1 \%$ aqueous ammonia was applied onto an ISOLUTE SLE $+400 \mu \mathrm{L}$ 96-well plate (Biotage) sitting on top of a clean 96-well collection plate. Positive pressure was applied for $2 \mathrm{~s}$ to initiate flow, 
and the sample was allowed to stand for 5 min to absorb into the diatomaceous earth extraction bed. Ethyl acetate $(700 \mu \mathrm{L})$ was added to the SLE array plate to elute the analytes. This elution process was repeated two more times.

The samples in the collection plate were evaporated to dryness under nitrogen at $40^{\circ} \mathrm{C}$. The residue was dissolved in 100 $\mu \mathrm{L}$ of $50 \%$ methanol and filtered using a $0.2-\mu \mathrm{m}$ spin filter. The filtrate was heated at $90^{\circ} \mathrm{C}$ for $30 \mathrm{~min}$ and then cooled at $4^{\circ} \mathrm{C}$ for a sufficient time using a thermal cycler. The solution after heat treatment was used as an analysis sample, and $10 \mu \mathrm{L}$ of sample was used for LC-MS/MS analysis. The sunitinib and $\mathrm{N}$-desethyl sunitinib samples were treated using a slightly modified method from that used for sunitinb $\mathrm{N}$-oxide. Briefly, the concentration of the ISTD was $1 \mu \mathrm{g} / \mathrm{mL}$, the residue was dissolved in 250 $\mu \mathrm{L}$ and the sample volume used for LCMS/MS analysis was $2 \mu \mathrm{L}$.

\subsection{Detection of sunitinib and metabolites}

A tandem quadrupole mass spectrometer was used to detect sunitinib and its metabolites. A Xevo-TQ (Waters) with ESI spray in positive ionization mode was used with the following ionization parameters: capillary voltage, $2.5 \mathrm{kV}$; desolvation temperature, $500^{\circ} \mathrm{C}$; source temperature, $150^{\circ} \mathrm{C}$; desolvation gas flow, $950 \mathrm{~L} / \mathrm{h}$; and cone gas flow, $50 \mathrm{~L} / \mathrm{h}$. The following transitions were monitored: 415.4/326.3 for sunitinib N-oxide, 399.4/283.2 for sunitinib, 371.4/283.2 for N-desethyl sunitinib, and 350.1/281.1 for voriconazole. The sample cone voltage and collision energy were $22 \mathrm{~V}$ and $20 \mathrm{~V}$ for sunitinib N-oxide, $30 \mathrm{~V}$ and $30 \mathrm{~V}$ for sunitinib, $22 \mathrm{~V}$ and $22 \mathrm{~V}$ for $\mathrm{N}$-desethyl sunitinib, and $20 \mathrm{~V}$ and $16 \mathrm{~V}$ for voriconazole, respectively. LC was performed with an ACQUITY UPLC. system (Waters) equipped with an ACQUITY UPLC BEH. C18 separation column $(2.1 \mathrm{~mm} \times 50 \mathrm{~mm}, 1.7 \mu \mathrm{m})$ (Waters). The LC conditions were as follows: column temperature, $40^{\circ} \mathrm{C}$; mobile phase, $10 \mathrm{mM}$ ammonium formate in MilliQ water (A) and $0.1 \%$ formic acid in acetonitrile (B); flow rate, $0.3 \mathrm{~mL} / \mathrm{min}$; and gradient program, $20 \%$ to $80 \% \mathrm{~B}$ in 3.0 min, $80 \%$ to $95 \% \mathrm{~B}$ in $0.1 \mathrm{~min}, 95 \% \mathrm{~B}$ for $0.2 \mathrm{~min}, 95 \%$ to $20 \% \mathrm{~B}$ in $0.2 \mathrm{~min}$, and $20 \%$ $\mathrm{B}$ for $1.5 \mathrm{~min}$.

\subsection{Assay validation}

Precision was measured as the coefficient of variation expressed as a percentage, and accuracy was expressed as the relative error of the nominal versus the measured concentration. The intra-assay variability was tested by measuring three different PHS samples against the same calibration curve. The inter-assay variability was tested on three different days and a new calibration curve was constructed for each day. The matrix effect was also evaluated quantitatively by measurement of the matrix factor.

The recovery ratio in extraction process was assessed by comparing quality control samples in blank PHS thatunderwent SLE with samples of the same concentration obtained by simply diluting the standard working solution.

The matrix factor was calculated by comparing the peak area of the sample obtained by spiking the compounds into SLE-treated blank PHS with that obtained by spiking the compound into $50 \%$ methanol. 


\section{Results}

\subsection{Detection of sunitinib and its metabolites}

Typical chromatograms of blank PHS and of blank PHS spiked with sunitinib Noxide, sunitinib, N-desethyl sunitinib, and voriconazole are shown in Figure 1.

\subsection{Validity of the calibration curve, accuracy and precision}

Good calibration curves were obtained from 0.1 to $5 \mathrm{ng} / \mathrm{mL}$ for sunitinib $\mathrm{N}$-oxide and $2.5-250 \mathrm{ng} / \mathrm{mL}$ for sunitinib and $\mathrm{N}$ desethyl sunitinib. For all three compounds, the weighting was set as $1 / \mathrm{x}^{2}$ and the

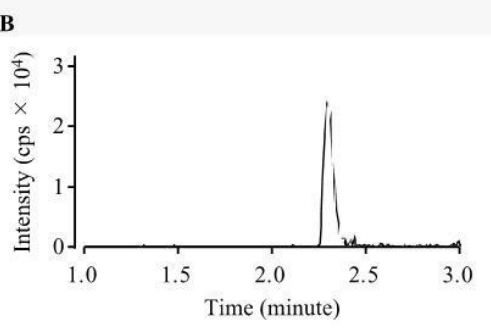

D

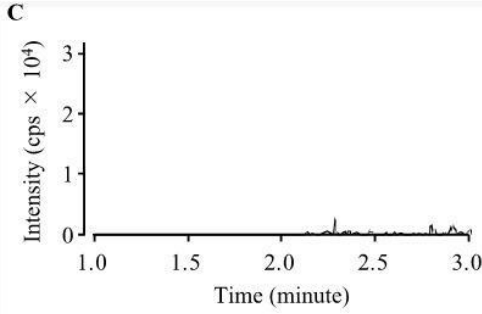

E
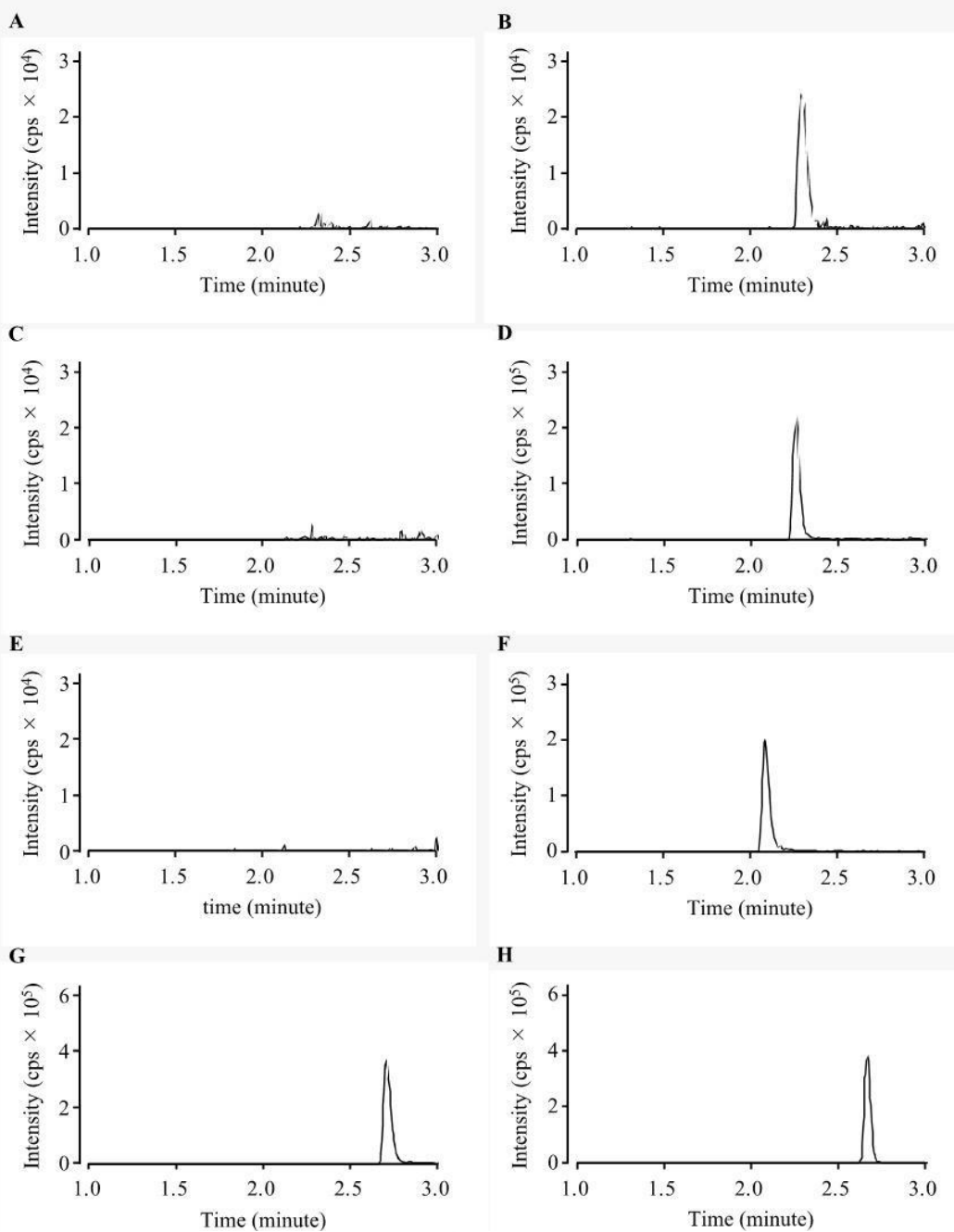

Figure 1 Typical chromatograms of sunitinib $\mathrm{N}$-oxide, sunitinib, $\mathrm{N}$-desethyl sunitinib, and voriconazole. A: sunitinib N-oxide (blank); B: sunitinib N-oxide ( $0.25 \mathrm{ng} / \mathrm{mL})$; C: sunitinib (blank); D: sunitinib (25 $\mathrm{ng} / \mathrm{mL})$; E: N-desethyl sunitinib (blank); F: N-desethyl sunitinib $(25 \mathrm{ng} / \mathrm{mL})$; G: voriconazole used as an ISTD for sunitinib N-oxide (10 $\mathrm{ng} / \mathrm{mL})$; H: voriconazole used as an ISTD for sunitinib and $\mathrm{N}$-desethyl sunitinib $(100 \mathrm{ng} / \mathrm{mL})$

The retention times of sunitinibNoxide, sunitinib, $\mathrm{N}$-desethyl sunitinib, and voriconazole were $2.25,2.26,2.09$, and $2.67 \mathrm{~min}$, respectively. The $E$-form of sunitinib and its metabolites, produced by photodecomposition reactions, were not detected. calibration curve was processed by quadratic curve fitting. The regression equation describing the calibration curve in PHS was $\mathrm{y}=2.81 \times 10^{-9} \mathrm{x}^{2}+0.000253 \mathrm{x}+$ $0.00202\left(\mathrm{R}^{2}=0.990\right)$ for sunitinib $\mathrm{N}$-oxide, $\mathrm{y}=9.81 \times 10^{-6} \mathrm{x}^{2}+0.0259 \mathrm{x}+0.00107\left(\mathrm{R}^{2}\right.$ $=0.995)$ for sunitinib, and $y=-4.94 \times 10^{-}$ ${ }^{7} \mathrm{x}^{2}+0.0235 \mathrm{x}+0.00107\left(\mathrm{R}^{2}=0.995\right)$ for $\mathrm{N}$ - 
desethyl sunitinib, where $y$ is the peak area ratio of the target compound against the ISTD and $\mathrm{x}$ is the nominal concentration of the target compound.
The results of accuracy and precision are shown in Tables 1 and 2.

Table 1 Inter-day validation

\begin{tabular}{|c|c|c|c|c|c|c|c|c|c|c|c|c|c|c|c|c|}
\hline \multirow{3}{*}{. } & \multirow{3}{*}{$\begin{array}{l}\text { Nominal } \\
\text { concentration } \\
(\mathrm{ng} / \mathrm{mL}) \\
0.1\end{array}$} & \multicolumn{5}{|c|}{ sunitinib N-oxide } & \multicolumn{5}{|c|}{ sunitinib } & \multicolumn{5}{|c|}{$\mathrm{N}$-desethyl sunitinib } \\
\hline & & \multicolumn{3}{|c|}{$\begin{array}{l}\text { Measured } \\
\text { concentration } \\
(\mathrm{ng} / \mathrm{mL})\end{array}$} & \multirow{2}{*}{$\begin{array}{l}\mathrm{Ac} \\
(\mathrm{RE} \%) \\
-2.35\end{array}$} & \multirow{2}{*}{$\begin{array}{l}\mathrm{Pr} \\
(\mathrm{CV} \%) \\
9.77\end{array}$} & \multicolumn{3}{|c|}{$\begin{array}{l}\text { Measured } \\
\text { concentration } \\
(\mathrm{ng} / \mathrm{mL})\end{array}$} & \multirow[t]{2}{*}{$\begin{array}{l}\text { Ac } \\
\text { (RE \%) }\end{array}$} & \multirow[t]{2}{*}{$\begin{array}{l}\operatorname{Pr} \\
(\mathrm{CV} \%)\end{array}$} & \multicolumn{3}{|c|}{$\begin{array}{l}\text { Measured } \\
\text { concentration } \\
(\mathrm{ng} / \mathrm{mL})\end{array}$} & \multirow[t]{2}{*}{$\begin{array}{l}\text { Ac } \\
\text { (RE \%) }\end{array}$} & \multirow[t]{2}{*}{$\begin{array}{l}\mathrm{Pr} \\
(\mathrm{CV} \%)\end{array}$} \\
\hline & & 0.098 & \pm & 0.010 & & & & & & & & & & & & \\
\hline & 0.25 & 0.270 & \pm & 0.017 & 8.13 & 6.24 & & & & & & & & & & \\
\hline \multirow{15}{*}{ 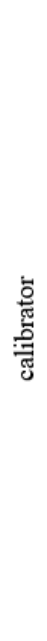 } & 0.5 & 0.489 & \pm & 0.057 & -2.30 & 11.6 & & & & & & & & & & \\
\hline & 1 & 0.949 & \pm & 0.045 & -5.13 & 4.74 & & & & & & & & & & \\
\hline & 2.5 & 2.54 & \pm & 0.22 & 1.70 & 8.79 & 2.49 & \pm & 0.18 & -0.33 & 7.35 & 2.51 & \pm & 0.21 & 0.33 & 8.41 \\
\hline & 5 & 5.00 & \pm & 0.46 & -0.04 & 9.22 & 5.09 & \pm & 0.36 & 1.83 & 6.98 & 4.97 & \pm & 0.48 & -0.67 & 9.69 \\
\hline & 10 & & & & & & 9.94 & \pm & 0.57 & -0.58 & 5.68 & 9.98 & \pm & 0.67 & -0.25 & 6.71 \\
\hline & 25 & & & & & & 24.9 & \pm & 1.42 & -0.23 & 5.69 & 25.5 & \pm & 1.88 & 2.03 & 7.38 \\
\hline & 50 & & & & & & 49.9 & \pm & 1.63 & -0.18 & 3.27 & 49.7 & \pm & 2.35 & -0.63 & 4.73 \\
\hline & 100 & & & & & & 99.7 & \pm & 4.36 & -0.28 & 4.37 & 98.8 & \pm & 4.63 & -1.25 & 4.69 \\
\hline & 250 & & & & & & 250 & \pm & 5.80 & 0.15 & 2.32 & 251 & \pm & 9.19 & 0.45 & 3.66 \\
\hline & 0.1 & 0.101 & \pm & 0.013 & 0.75 & 13.2 & & & & & & & & & & \\
\hline & 0.25 & 0.274 & \pm & 0.026 & 9.77 & 9.48 & & & & & & & & & & \\
\hline & 1 & 1.03 & \pm & 0.110 & 3.00 & 10.6 & & & & & & & & & & \\
\hline & 2.5 & & & & & & 2.88 & \pm & 0.19 & 15.3 & 6.59 & 2.78 & \pm & 0.27 & 11.0 & 9.73 \\
\hline & 5 & 5.18 & \pm & 0.702 & 3.68 & 13.6 & 5.18 & \pm & 0.35 & 3.67 & 6.78 & 5.03 & \pm & 0.40 & 0.67 & 8.01 \\
\hline & 50 & & & & & & 51.5 & \pm & 2.95 & 3.00 & 5.74 & 50.9 & \pm & 3.51 & 1.83 & 6.88 \\
\hline$\ddot{\partial}$ & 250 & & & & & & 259 & \pm & 10.4 & 3.53 & 4.01 & 263 & \pm & 12.8 & 5.19 & 4.88 \\
\hline
\end{tabular}

Ac, accuracy; $\operatorname{Pr}$, precision

Table 2 Intra-day validation

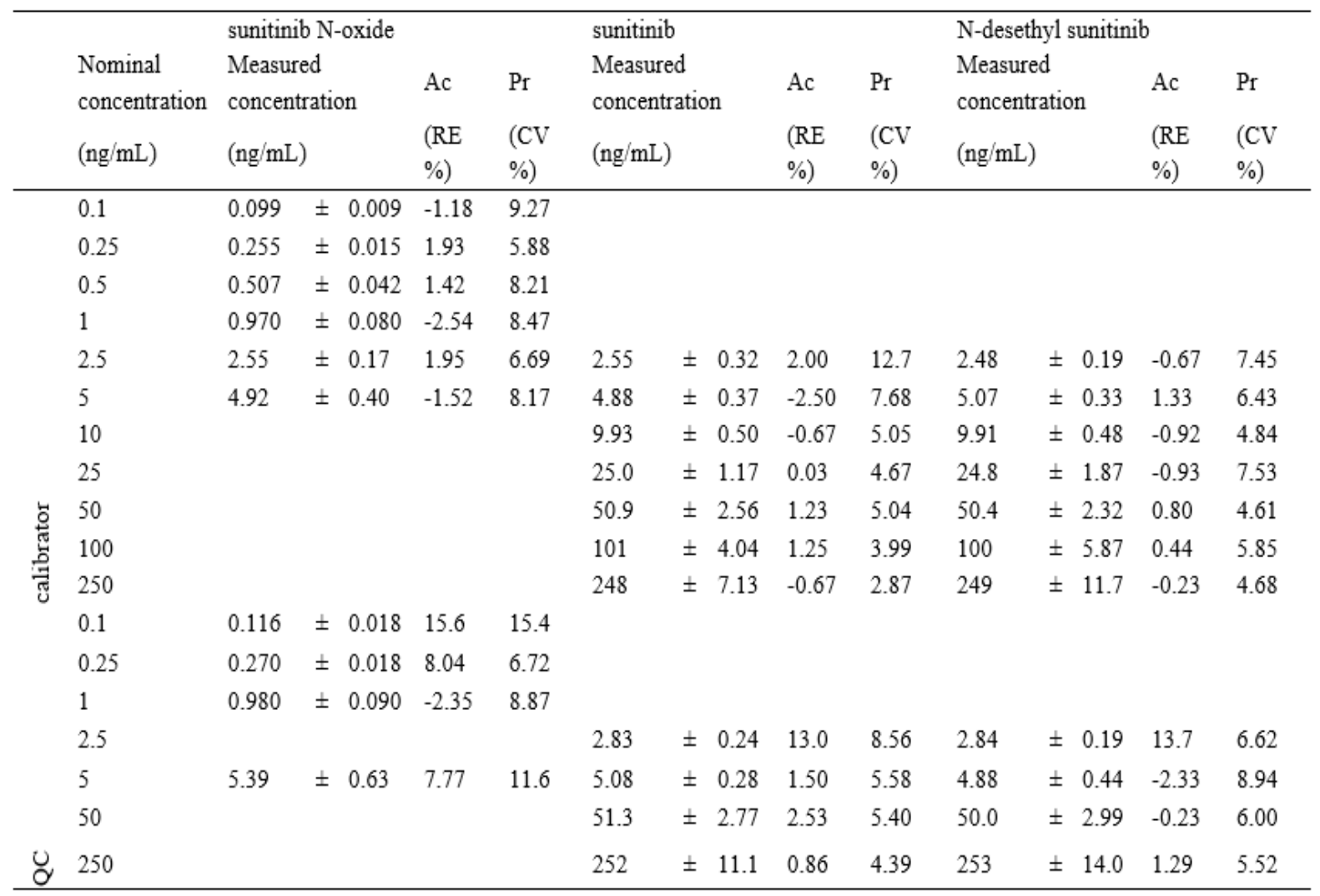

accuracy; $\mathrm{Pr}$, precision 


\subsection{Extraction recovery}

The recovery of sunitinib $\mathrm{N}$-oxide and ISTD prior to SLE treatment for the assay of sunitinib $\mathrm{N}$-oxide and the recovery of

sunitinib, N-desethyl sunitinib and ISTD prior to SLE treatment for the assay of sunitinib and N-desethyl sunitinib are shown in Table 3. All recoveries were $>80 \%$.

\subsection{Interference and matrix effect}

The relative standard deviation was less than $15 \%$ and peaks corresponding to sunitinib and its metabolites were not detected in the blank PHS sample. The matrix factors are shown in Table 3, suggesting that no significant matrix effect was observed and thus had no adverse impact on the quality of the data.

\section{Discussion}

Sunitinib N-oxide has been the urine of animals such as rats, human blood [22]. Therefore, the effects of sunitinib N-oxide on the human body have not been investigated well, and to date there is no analytical method for sunitinib $\mathrm{N}$-oxide in blood. Previously, we found that sunitinib $\mathrm{N}$-oxide, a photodegradant of sunitinib, is found in the blood of patients taking sunitinib [17], and to clarify the effect of sunitinib N-oxide on the onset of ADRs associated with sunitinib, we developed a simple method for measuring the sunitinib $\mathrm{N}$-oxide concentration in blood. We previously reported that the concentration of sunitinib $\mathrm{N}$-oxide is estimated to be about $1 / 20$ that of sunitinib [17], so it was difficult to apply the measurement method of sunitinib directly to analysis of sunitinib $\mathrm{N}$-oxide, and therefore a more sensitive method than previously reported methods used for sunitinib is needed. Here, we used sample enrichment as a pretreatment and an LC-MS/MS method for analyte detection, and developed a method for measurement of sunitinib $\mathrm{N}$-oxide with good calibration curve $\left(\mathrm{R}^{2} \geqq 0.99\right)$. This is a first report on the measurement method of sunitinib $\mathrm{N}$ oxide.

Sunitinib is light-unstable and converts from the $Z$ form to the $E$ form upon light exposure [23]. The blood concentration of sunitinib (typically $Z$-sunitinib) may be underestimated because of generation of the $E$ form, and the sum of the $E$ and $Z$ form concentrations is generally evaluated as the sunitinib concentration [24]. However, since the ionization efficiency at the time of elution of the $E$ and $Z$ forms may differ upon LC separation using a gradient method, as is typical in analyses using MS/MS, the sum of the concentrations of the $E$ and $Z$ forms cannot be evaluated by simply adding the obtained peak areas. In addition, there is currently no technique for maintaining the $E$ and $Z$ forms separately in a liquid sample, and therefore we cannot perform accurate absolute quantification using these standard products. This issue was addressed by converting the $E$ form to the $Z$ form by heat-treating the sample according to the reports by Posocco et al. [25] and Marangon et al. [26]. Although details of the conversion efficiency from the $E$ to the $Z$ form by heating have not been clarified, at least in the analysis of sunitinib N-oxide using our method, no $E$ form of sunitinib $\mathrm{N}$-oxide was detected, and no sunitinib $E$ form was also detected when analyzed using our method, which can detect sunitinib at concentrations 20 times lower than the lower limit of detection of methods used in previous reports (data not shown). In addition, the $E$ form in samples at the time of LC injection is estimated to be less than $0.1 \%$ of each sunitinib 
concentration used in our study, indicating that the $E$ form is converted to the $Z$ form with sufficient efficiency.

A deproteinization pretreatment method is typically used to analyze sunitinib using LC-MS/MS. However, since a sample enrichment is needed to analyze sunitinib $\mathrm{N}$-oxide as described above, the SLE method was adopted to pretreat the samples in this study. The SLE method has been used to measure many drugs because of its ease and rapidity but details of its application to sunitinib and its metabolites have not been reported. 
Therefore, in this study, we evaluated in detail the applicability of the SLE method to the LC-MS/MS analysis of sunitinib and its metabolites and confirmed the utility of this approach. SLE method has a higher capacity to remove proteins from serum and can accurately recover the target components. In addition, the solid-phase extraction method requires washing after fixation of the target components on the solid phase, which requires many steps, while the SLE method does not require washing, thus shortening the working time and achieving high recovery ratio. Due to these characteristics, the pretreatment method using SLE is not easily affected by the skills of technicians and can be said to be one of the methods suitable for clinical application. Furthermore, SLE likely increases the purity of the samples injected onto LC-MS/MS, facilitating the analysis of a large number of samples.

In addition, we tried to separate the peaks corresponding to sunitinib and sunitinib $\mathrm{N}$-oxide by LC but complete separation was not achieved in the short time analysis. Although many LC-UV methods have been reported for measuring sunitinib and $\mathrm{N}$-desethyl sunitinib concentrations [19], these compounds cannot be distinguished by UV, raising the possibility of overestimating the concentration of sunitinib by LC-UV analysis. Therefore, LC-UV is not amenable to rapid analysis of the concentrations of sunitinib and its metabolites and an LC-MS/MS method is recommended.

In conclusion, we reported the first report on the method for analyzing the blood concentration of sunitinib N-oxide, a sunitinib metabolite in the human body. Our analysis method for of sunitinib Noxide, sunitinib and $\mathrm{N}$-desethyl sunitinib is simple and the analytes are made more stable by pretreatment using an SLE method and heat treating the sample. Since our method requires only simple steps and a column, it is not easily affected by variations in operator skill. We believe that the method reported here will facilitate understanding of the effects of sunitinib $\mathrm{N}$ oxide in humans, contribute to elucidation of the ADR expression factors associated with sunitinib, and aid in optimizing treatment with sunitinib.

\section{Reference}

[1] James L, Abby P, Grace F, Stephen $\mathrm{M}$, Connie C. First-line treatment in the management of advanced renal cell carcinoma: systematic review and network meta-analysis. Expert Opin Pharmacother. 2015;16(13):1915-27.

[2] Olivia LS, Gilles F, Sylvie N. FirstLine Treatments for Poor-Prognosis Metastatic Renal Cell Carcinoma: Experts' Prescribing Practices and Systematic Literature Review. Clin Drug Investig. 2016;36(5):389-99.

[3] Cancel M, Fromont G, Blonz C, Chevreau C, Rioux-Leclercq N, Laguerre $\mathrm{B}$, et al. A retrospective study of the GETUG group (Groupe d'Etude des Tumeurs UroGénitales). Eur. J. Cancer. 2021;158(4):1-11.

[4] Hao Z, Sadek I. Sunitinib: The antiangiogenic effects and beyond. Onco. Targets. Ther. 2016;9(8):5495-505.

[5] Lim S, Hwang I, Ji J, Oh S, Yi J, Lim $\mathrm{D}$, et al. Intrinsic resistance to sunitinib in patients with metastatic renal cell carcinoma. Asia. Pac. J. Clin. Oncol. 2017;13(1):61-67. 
[6] Gore M, Szczylik C, Porta C, Bracarda S, Bjarnason G, Oudard S, et al. Final results from the large sunitinib global expanded-access trial in metastatic renal cell carcinoma. $\mathrm{Br} \quad \mathrm{J}$ Cancer. 2015;113(1):12-9.

[7] Takasaki S, Kawasaki Y, Kikuchi M, Tanaka M, Suzuka M, Noda A, et al. Relationships between sunitinib plasma concentration and clinical outcomes in Japanese patients with metastatic renal cell carcinoma. Int. J. Clin. Oncol.2018;23(5):936-43.

[8] Westerdijk K, Krens S, Graaf W, Mulder S, Herpen C, Smilde T, et al. The relationship between sunitinib exposure and both efficacy and toxicity in real-world patients with renal cell carcinoma and gastrointestinal stromal tumour. Br. J. Clin. Pharmacol. 2021;87(2): 32635.

[9] Noda S, Otsuji T, Baba M, Yoshida T, Kageyama S, Okamoto K, et al. Assessment of Sunitinib-Induced Toxicities and Clinical Outcomes Based on Therapeutic Drug Monitoring of Sunitinib for Patients with Renal Cell Carcinoma. Clin. Genitourin.

Cancer. 2015;13(4):350-8.

[10] Adams V, Leggas M. Sunitinib Malate for the Treatment of Metastatic Renal Cell Carcinoma and Gastrointestinal Stromal Tumors. Clin. Ther. 2007;29(7):1338-53.

[11] Werbrouck E, Bastin J, Lambrechts D, Verbiest A, Thomas Van Brussel, Lerut E, et al. ABCG2 Polymorphism rs2231142 and hypothyroidism in metastatic renal cell carcinoma patients treated with sunitinib. Acta Clin. Belg. 2019;74(3):180-8.

[12] Mizuno T, Fukudo M, Terada T, Kamba T, Nakamura E, Ogawa O, et al. Impact of genetic variation in breast cancer resistance protein (BCRP/ABCG2) on sunitinib pharmacokinetics. Drug Metab. Pharmacokinet. 2012;27(6):631-9.

[13] Laura W, Stephanie T, Lufang Y, Dustin L. Real-World Use of Azathioprine Metabolites Changes Clinical Management of Inflammatory Bowel Disease. J Can Assoc Gastroenterol. 2020;4(3):101-9.

[14] Georgios S, Benedikt S, Christoph H, Gerhard G, Koen RS, Sebastian $\mathrm{W}$, et al. Pharmacokinetic patterns of risperidone-associated adverse drug reactions. Eur J Clin Pharmacol. 2016;72(9):1091-8.

[15] Takahiro Y, Yasuaki M, Tatsuya Y, Takafumi N, Junichi K. Saturated Metabolism of Voriconazole NOxidation Resulting in Nonlinearity of Pharmacokinetics of Voriconazole at Clinical Doses. Biol Pharm Bull. 2015;38(10):1496-503.

[16] Shimada M, Okawa H, Kondo Y, Maejima T, Kataoka Y, Hisamichi K, et al. Monitoring serum levels of sorafenib and its $\mathrm{N}$-oxide is essential for long-term sorafenib treatment of patients with hepatocellular carcinoma. Tohoku J. Exp. Med. 2015;237(3):173-82.

[17] Takenaka M, Takahashi Y, Yashima $\mathrm{H}$, Araki T, Yamamoto K. The Impact of Sunitinib N-oxide as a Photodegradation Product of Sunitinib. Indones. J. Pharm. 2019;1(1):19-25. 
[18] Amemiya T, Honma M, Kariya Y, Ghosh S, Hiroaki Kitano H, Kurachi Y., Elucidation of the molecular mechanisms underlying adverse reactions associated with a kinase inhibitor using systems toxicology. NPJ Syst Biol Appl. 2015;1(28):110.

[19] Grimaldi M, Renée N, Izzedine H, Milano G. A routine feasible HPLC analysis for the anti-angiogenic tyrosine kinase inhibitor, sunitinib, and its main metabolite, SU12662, in plasma. J. Chromatogr. B Anal. Technol. Biomed. Life Sci. 2009;877(29):3757-61.

[20] Erp N, Wit D, Guchelaar H, Gelderblom H, Hessing T, Hartigh J. A validated assay for the simultaneous quantification of six tyrosine kinase inhibitors and two active metabolites in human serum using liquid chromatography coupled with tandem mass spectrometry. J. Chromatogr. B Anal. Technol. Biomed. Life Sci. 2013;937(15):33-43.

[21] Takasaki S, Tanaka M, Kikuchi M, Maekawa M, Kawasaki Y, Ito A. Simultaneous analysis of oral anticancer drugs for renal cell carcinoma in human plasma using liquid chromatography/electrospray ionization tandem mass spectrometry. Biomed. Chromatogr. 2018;32(6):1-8.

[22] Bill S, Hai-Zhi B, William P, Geoffrey P, Ellen W, Shem P, et al. Carlo B, Ping K. Pharmacokinetics, distribution, and metabolism of [14C]sunitinib in rats, monkeys, and humans. Drug Metab Dispos. 2012;40(3):539-55.
[23] Matsunaga N, Kitahara T, Yamada M, Sato K, Kodama Y, Sasaki H. The influence of light sources on sunitinib measurements with photoisomerization. Biomed. Chromatogr. 2019;33(2):e4407.

[24] Haouala A, Zanolari B, Rochat B, Montemurro M, Zaman K, Duchosal $\mathrm{M}$, et al. Therapeutic Drug Monitoring of the new targeted anticancer agents imatinib, nilotinib, dasatinib, sunitinib, sorafenib and lapatinib by LC tandem mass spectrometry. Journal of Chromatography B. 2009;877(22):1982-96.

[25] Posocco B, Buzzo M, Giodini L, Crotti S, D'Aronco S, Trald P. Analytical aspects of sunitinib and its geometric isomerism towards therapeutic drug monitoring in clinical routine. J. Pharm. Biomed. Anal.2018;160(25):360-7.

[26] Marangon E, Buzzo M, Posocco B, Gagno $S$, Zanchetta $M$, Iacuzzi $V$, et al. A new high-performance liquid chromatography-tandem mass spectrometry method for the determination of sunitinib and $\mathrm{N}$ desethyl sunitinib in human plasma: Light-induced isomerism overtaking towards therapeutic drug monitoring in clinical routine. J. Pharm. Biomed. Anal.2020;179(5):112949. 